\title{
Cost-effectiveness of patient navigation for breast cancer screening in the National Breast and Cervical Cancer Early Detection Program
}

\author{
Benjamin T. Allaire ${ }^{1}$, Donatus Ekweme ${ }^{2}$, Thomas J. Hoerger ${ }^{1}$, Amy DeGroff $^{2}$, Sun Hee \\ Rim $^{2}$, Sujha Subramanian ${ }^{1}$, Jacqueline W. Miller ${ }^{2}$ \\ ${ }^{1}$ RTI International, 3040 E. Cornwallis Road, P.O. Box 12194, Research Triangle Park, NC 27709, \\ USA \\ ${ }^{2}$ Centers for Disease Control and Prevention, Atlanta, GA 30341, USA
}

\begin{abstract}
Objectives-Patient navigation (PN) services have been shown to improve cancer screening in disparate populations. This study estimates the cost-effectiveness of implementing PN services within the National Breast and Cervical Cancer Early Detection Program (NBCCEDP).

Methods-We adapted a breast cancer simulation model to estimate a population cohort of women aged 40-64 years from the NBCCEDP through their lifetime. We incorporated their screening frequency and screening and diagnostic costs.
\end{abstract}

Results-Within the NBCCEDP, Program with PN (vs. No PN) resulted in a greater number of mammograms per woman (4.23 vs. 4.14), lower lifetime mortality from breast cancer (3.53\% vs. $3.61 \%$ ), and fewer missed diagnostic resolution per woman (0.017 vs. 0.025 ). The estimated incremental cost-effectiveness ratios for a Program with PN was $\$ 32,531$ per quality-adjusted lifeyears relative to Program with No PN.

Conclusions-Incorporating PN services within the NBCCEDP may be a cost-effective way of improving adherence to screening and diagnostic resolution for women who have abnormal results from screening mammography. Our study highlights the value of supportive services such as PN in improving the quality of care offered within the NBCCEDP.

\section{Keywords}

Breast cancer; Screening; Mammography; Cost-effectiveness

\section{Introduction}

\begin{abstract}
About one in four cancers diagnosed in women are breast cancers [1]. Breast cancer is the most commonly diagnosed cancer and the second leading cause of death among women in the U.S [1]. Each year, about 220,000 diagnoses and about 40,000 breast cancer deaths
\end{abstract}

Benjamin T. Allaire, ballaire@ @ri.org.

Publisher's Note Springer Nature remains neutral with regard to jurisdictional claims in published maps and institutional affiliations. 
occur [1]. The U.S. Preventive Services Task Force (USPSTF), the American Cancer Society, and several medical societies all recommend screening for breast cancer using either annual or biennial mammograms; [2, 3] however, data from the 2015 National Health Interview Survey show that mammography rates have remained relatively stable from 2000 to $2015,71.5 \%$ of women aged 50-74 years reporting having had a mammogram in the past 2 years [4]. Despite the known benefits of regular breast cancer screening in reducing morbidity and mortality through early detection and follow-up care, mammography use varies by subpopulations and is lowest among those who report being uninsured or without a usual source of healthcare [4].

The Centers for Disease Control and Prevention's (CDC) National Breast and Cervical Cancer Early Detection Program (NBCCEDP), which currently operates in all 50 states, the District of Columbia, 6 U.S. territories, and 13 American Indian and Alaska Native tribal jurisdictions, was established by the U.S. Congress in 1990 to provide breast and cervical cancer screening to uninsured and underinsured low-income women. The program also gives awardees resources to educate the public and healthcare providers about screening for breast and cervical cancer and to conduct outreach activities that identify underserved women who are in need of screening services. Since its inception, the NBCCEDP has provided more than 12.7 million breast and cervical cancer screening tests to more than 5.3 million low-income women [5]. Even with adequate health insurance and coverage of clinical services, studies suggest that many women still face significant barriers in obtaining breast cancer screening due to language barriers, low health literacy, geographic isolation, transportation cost to provider office, inconvenient appointment times to access services, lack of paid sick leave, lack of education about cancer screening and screening tests, and fear, mistrust of the medical system [6-10].

Implementation of patient navigation (PN) services has been shown to reduce some of the barriers experienced by women and improve compliance with breast cancer screening [11, 12]. Navigation provided to patients in the form of education, language translation services, reimbursement for transportation, guidance in interpreting doctor recommendations, emotional support, and help completing required documents have been used by NBCCEDP programs to reduce barriers related to undergoing screening and diagnostic testing [13]. Systematic establishment of PN services could also help increase screening compliance among insured women by eliminating barriers [13].

To date, there have been limited economic assessments of the NBCCEDP [14-17] and no systematic studies conducted on the cost and effectiveness of the program's PN activities. In an accompanying article, we estimate the overall cost-effectiveness of breast cancer screening in the NBCCEDP [18]. In this study, we expand upon the prior analysis to assess the cost-effectiveness of PN services within the NBCCEDP using a hybrid decision analyticsimulation model. We expect that these findings will enable the NBCCEDP awardees to assess the cost and effectiveness of implementing PN to reduce barriers faced by women when undergoing breast cancer screening and diagnostic testing.

Cancer Causes Control. Author manuscript; available in PMC 2019 October 01. 


\section{Methods}

\section{Model description}

A detailed description of the cost-effectiveness model for this paper is described in the accompanying article and in Hoerger et al. [15]. Briefly, we modified a validated breast cancer screening simulation model developed by the Cancer Intervention and Surveillance Modeling Network (CISNET) [19-21]. We added data from the NBCCEDP on patient cohorts, screening frequency, and program costs, and calculated life-time costs and qualityadjusted life-years (QALYs). We calculated the costs and QALYs for four scenarios: (1) breast cancer screening in the NBCCEDP; (2) less frequent screening of low-income, noninsured women that would take place in the absence of the program [i.e. "No Program"]; (3) breast cancer outcomes that would occur if no screening took place within the program since clinical detection would still occur under this scenario [i.e. "No Screening"]; and (4) PN services that would occur within the NBCCEDP [i.e. "Program with PN"]. The NBCCEDP scenarios (Program with PN and No PN scenarios) includes costs associated with breast cancer screening and program delivery. More details on the costs included in the Program with PN and No PN scenarios are available in Rim et al. [18]. We estimated the incremental costs and QALYs for each scenario and calculated incremental cost-effectiveness ratios (ICERs) for the NBCCEDP relative to the other scenarios. The model takes a societal perspective.

\section{Impact of PN on cancer screening}

We reviewed published studies on PN in breast cancer screening and identified three areas where navigation is likely to improve patient care: adherence to screening, completion of diagnostic resolution, and timeliness of diagnostic follow-up. Table 1 shows our model assumptions in these areas. Wells et al. found that the rate of adherence to screening increased between $10.8 \%$ and $17.1 \%$ when patients in a navigation group were compared with a control group [22]. We modelled this effect as a reduction of 14\% (the midpoint of the estimated range in Wells et al. in the share of irregular screeners in the NBCCEDP in our analysis. Our model contained three screening types: annual, biennial, and irregular. Annual and biennial screeners were considered those who adhered to screening recommendations. In the NBCCEDP, the percentage of irregular screeners ranged from 9\% (age 60-65) to 28\% (age 40) (see Hoerger et al. for details on this calculation) [15]. For comparison, in lowincome, uninsured women not covered by the program, the percentage of irregular screeners ranged from $47 \%$ (age 60-65) to 64\% (age 40) [15].

For diagnostic resolution, the evidence on PN is mixed. Battaglia et al. found a slight increase in complete diagnostic follow-up in their unadjusted results and a statistically significant hazard ratio for diagnostic resolution after 60 days [23]. Although Clark et al. found an increase in diagnostic follow-up, this could not be attributed to the intervention [24]. Markossian et al. reported high unadjusted rates for diagnostic adherence (98.7\% for the PN arm versus $81 \%$ for the control group) [25], but the results were not significantly associated with improved odds of diagnostic resolution after adjusting for covariates. 
In contrast, several studies have shown PN to be effective in improving diagnostic resolution $[12,26,27]$. For example, Raich and colleagues found $92 \%$ of the navigated patients reached diagnostic resolution of the initial abnormal test, as compared with $77 \%$ for the control patients $(P<0.001)$ [12]. In addition, Battaglia et al. found statistically significant improvements in diagnostic adherence: $65 \%$ of pre-intervention versus $76 \%$ of intervention subjects had timely follow-up $(P=0.008)$ [26]. Further, Ferrantes et al. showed, in a small group, that $94 \%$ of patients with navigation completed diagnostic follow-up versus $78 \%$ in the control [27]. In the present study, we assumed a baseline rate of $65 \%$ diagnostic adherence [26] for those not in the program and an improvement to $90 \%$ diagnostic adherence within the program based on expert opinion of NBCCEDP data and the results from Miller et al. [28]. This is a substantial improvement that is probably not solely due to PN services. Therefore, we assumed a more modest increase in diagnostic follow-up (from 77 to $92 \%$ ) based on a study by Raich et al. [12].

Several studies also have demonstrated that PN reduces time to diagnostic follow-up [22, 27, 29]. Based on prior studies, we introduced a time delay for navigated and non-navigated women in the program of 25 and 43 days, respectively [22, 27].

\section{Costs of PN}

PN costs were obtained from a survey of the subcontractors participating in Colorado's "Connect-to-Care" component of the state's NBCCEDP-funded program in 2011 (Personal Communication, Christen Lara 2013; Connect to Health 2014). Forty-two of the state's 45 subcontractors participated in the survey, which measured the time spent for a patient navigator for screening and also the time spent for navigation after an abnormal screening result. The survey found that PN was conducted by non-clinical staff typically for screening and clinical staff after an abnormal screening result. Respondents indicated that half of patients received navigation for screening and that about $95 \%$ of the time navigation took less than an hour. Navigating for diagnostic resolution after an abnormal screen result, however, typically took between 30 and 90 min to complete. We estimated a cost of PN to be $\$ 8.52$ per screen and $\$ 30.53$ per abnormal screen result. Using the hourly rate provided by the state of Colorado and inflated to 2018 costs we calculated the following hourly ratesnonclinical (\$14.80) and clinical staff (\$26.50). We assume 30-mins of a patient navigator for screening and an hour for diagnostic resolution.

\section{Outcomes}

The main outcomes for each scenario were: estimates of screening history, breast cancer incidence, screening or clinical detection, stage at detection, costs, life-years, and QALYs. Costs and QALYs were summed for each scenario. The effect of PN in the NBCCEDP was estimated as the difference in costs and the difference in QALYs between the Program with PN and No PN scenarios. The ICERs were then estimated by dividing the difference in costs by the difference in QALYs. We also compared the Program with PN and No PN (also within the NBCCEDP) to the No Program and No Screening scenarios described in the accompanying article. The model was developed and analyzed in TreeAge Pro 2009 (TreeAge Software Inc, Williamstown, MA). Costs are reported in 2018 U.S. dollars; costs and QALYs were discounted at a 3\% annual rate.

Cancer Causes Control. Author manuscript; available in PMC 2019 October 01. 


\section{Sensitivity analyses}

Sensitivity analyses for the model involved running deterministic and probabilistic sensitivity analyses (PSA). The results of all parameters are presented in Rim et al. of the sensitivity analyses for the model [18]. For this study, we ran an additional sensitivity analyses varying the total costs of $\mathrm{PN}$ by $\pm 50 \%$.

\section{Results}

Results from the four simulations estimated breast cancer mortality as $3.53 \%, 3.61 \%$, $3.93 \%$, and $4.97 \%$ under the respective scenarios of Program with PN, No PN, No Program, and No Screening scenarios (Table 2). In terms of screen-detected cancers, the model estimated a modest increase for Program with PN compared to Program without PN (8.55\% vs. $8.25 \%$ ). Existence of the program, as demonstrated in both scenarios, resulted in estimated 1.69 to 1.99 percentage point increases in screen-detected cancers relative to the No Program scenario $(6.56 \%)$. Women were estimated to have fewer missed diagnostic follow-ups with PN (0.017/woman) compared to the No PN scenario (0.025/woman).

Overall costs per woman in the modeled cohort were higher in the Program with PN, No PN, and No Program scenarios than in the No Screening scenario. Screening costs accounted for the largest share of the differences between scenarios; treatment costs were also higher in the Program with PN, No PN, and No Program scenarios compared to the No Screening scenario. Diagnostic costs were higher in the Program with PN scenario than in the No PN scenario, reflecting the decrease in missed follow-ups. Navigation costs were \$126 per woman in the Program with PN scenario.

We estimated that including navigation in the Program would lead to an increase of 0.006, 0.024 and 0.071 QALYs relative to the No PN, No Program, and No Screening scenarios, respectively. Incremental (discounted) life-years were slightly higher. The Program with PN had an ICER of \$33,600 per QALY and \$51,120 per QALY relative to the No PN and No Program scenarios, respectively. Relative to either the No Program or No Screening scenarios, the Program with PN had lower ICERs than the No PN scenarios (Table 2). In cost-effectiveness terms, the Program with PN scenario has extended dominance over the No PN scenario, because the former scenario provided more QALYs with a lower ICER.

We also calculated ICERs based on discounted life-years gained. Estimates of life-years gained do not take into account the quality-of-life decrements included in the QALY estimates. Therefore, because estimated life-years gained exceeded QALYs gained, the lifeyear ICERs were slightly lower than the QALY ICERs.

PN costs have a significant impact on the ICER. When we decrease patient navigation costs by $50 \%$, we find the Program with PN ICER drops to $\$ 23,136$ per QALY relative to the No PN scenario. Increasing costs increases the Program with PN ICER to \$44,064. (Results available on request.) 


\section{Discussion}

Based on our simulations, PN within the NBCCEDP-improved outcomes but also raised costs. The estimated ICER is considered cost-effective by some thresholds at $\$ 33,600$ per QALY relative to No PN. In the accompanying paper, we report that the ICER for the NBCCEDP as a whole (including PN) was $\$ 51,120$ and $\$ 49,596$ per QALY relative to scenarios with less frequent or no screening, respectively. If incorporating PN into NBCCEDP improves screening frequency for irregular screeners and increases diagnostic resolution rates for women who have abnormal screening results, our results suggest that the program may be cost-effective.

The PN became a required NBCCEDP activity in 1999 when Congress appropriated specific funds to support it. For low income, un- and under-insured women who face multiple personal and health system barriers, PN provides the necessary support and resources to help them complete screening and diagnostic follow-up. Navigation has also been shown to decrease disparities in breast cancer outcomes [30-33] and increase patient satisfaction [34]. The CDC continues to recognize the value of $\mathrm{PN}$ and has expanded the service beyond traditionally program-eligible women to include low-income women who have other resources for mammography, including those recently insured through expanded health care under the Affordable Care Act. Data suggest that among age-appropriate women with public insurance, 23-29\% remain unscreened or not up to date with screening [4]. CDC encouraged awardees to use PN to link those women in the community who have never been screened or do not have a medical home to cancer screening. Without reaching these populations, breast cancer screening rates are likely to continue at current levels, which is below the Healthy People 2020 target [4].

Our analysis is subject to certain limitations. First, $\mathrm{PN}$ is one part of a more comprehensive NBCCEDP health promotional activity, so it is difficult to precisely estimate the impact of the navigation services within the program. We use assumptions about the impact of PN from studies of NBCCEDP non-participants; as such, results may not be generalizable to the NBCCEDP. On the other hand, these studies have focused on vulnerable populations, including low-income, uninsured women who are the target population for the NBCCEDP. Second, while using data from an actual NBCCEDP awardees (i.e., Colorado) is a strength of this study, we recognize that PN costs likely vary across NBCCEDP awardee in different states. Third, long-term breast cancer treatment outcomes are based on a simulation model that relates PN to screening and diagnostic follow-up, and then relates the screening and diagnostic follow-up to breast cancer outcomes. Although a study directly linking observed PN services to observed breast cancer outcomes would provide more compelling evidence on the impact of PN, such a study is not likely to be feasible because it would require a long follow-up period and a large sample size. As a more feasible alternative approach, simulation models link an intervention's short-term outcomes to long-term impact. Fourth, our cost estimates reflect the direct costs of PN and do not take into account overhead costs when sourcing the costs for the PN program. Sensitivity analysis results around this estimate by increasing by $50 \%$ found that $\mathrm{PN}$ is still cost-effective relative to No PN. 
Our study has potential implications for breast cancer screening among low-income women. The Affordable Care Act (ACA) expanded health insurance coverage to previously uninsured low-income women (through health insurance exchanges or Medicaid coverage in states that expand their programs), improving access to preventive services, including breast cancer screening. This expanded coverage, particularly to low-income populations, may not be enough to ensure that beneficiaries actually receive cancer screening. Therefore, PN may be essential to ensure that these women get appropriate and complete screening and diagnostic services as reported in this paper and in other studies [22, 25, 35, 36]. As our results suggest, providing PN may be cost-effective if it can be provided at costs that are similar to those in the NBCCEDP. Even with the availability of health insurance, some lowincome women will still remain uninsured [35]. The estimates reported in this paper indicate that incorporating PN activities in the NBCCEDP breast cancer screening and diagnostic services could be a cost-effective way of improving adherence to cancer screening in medically underserved low-income women.

\section{Acknowledgments}

We would like to thank Christen Lara from the Colorado Department of Public Health and the Environment for her assistance in compiling Colorado NBCCEDP cost data for this manuscript.

Funding This research was supported by Contract No. 200-2008-27958 TO 27 from the Centers for Disease Control and Prevention (CDC). The findings and conclusions in this report are those of the authors and do not necessarily represent the official position of the CDC.

\section{References}

1. U.S. Cancer Statistics Working Group (2017) United States Cancer Statistics: 1999-2014 Incidence and Mortality Web-based Report. Atlanta: U.S. Department of Health and Human Services, Centers for Disease Control and Prevention and National Cancer Institute www.cdc.gov/uscs

2. Siu AL USPSTF (2016) Screening for breast cancer: U.S. Preventive Services Task Force Recommendation Statement. Ann Intern Med 164(4):279-296 [PubMed: 26757170]

3. Oeffinger KC, Fontham ET, Etzioni R et al. (2015) Breast cancer screening for women at average risk: 2015 guideline update from the American Cancer Society. JAMA 314(15):1599-1614 [PubMed: 26501536]

4. White A, Thompson TD, White MC, Sabatino SA, de Moor J, Doria-Rose PV, Geiger AM, Richardson LC (2017) Cancer screening test use-United States, 2015. MMWR Morb Mortal Wkly Rep 66(8):201-206. 10.15585/mmwr.mm6608a1 [PubMed: 28253225]

5. CDC Division of Cancer Prevention and Control. National Breast and Cervical Cancer Early Detection Program (NBCCEDP), About the Program, Accomplishments [Internet]. CDC Cancer Division www.cdc.gov/cancer/nbccedp/about.htm

6. Bhanegaonkar A, Madhavan SS, Khanna R, Remick SC (2012) Declining mammography screening in a state Medicaid Feefor-Service program: 1999-2008. J Womens Health (Larchmt). 21(8):821829 [PubMed: 22568434]

7. Peipins LA, Graham S, Young R, Lewis B, Foster S, Flanagan B, Dent A (2011) Time and distance barriers to mammography facilities in the Atlanta metropolitan area. J Community Health 36(4): 675-683 [PubMed: 21267639]

8. Alexandraki I, Mooradian AD (2010) Barriers related to mammography use for breast cancer screening among minority women. J Natl Med Assoc 102(3):206-218 [PubMed: 20355350]

9. Davis JL, Bynum SA, Katz RV, Buchanan K, Green BL (2012) Sociodemographic differences in fears and mistrust contributing to unwillingness to participate in cancer screenings. J Health Care Poor Underserved 23(4 Suppl):67-76 
10. Peipins LA, Soman A, Berkowitz Z, White MC (2012) The lack of paid sick leave as a barrier to cancer screening and medical care-seeking: results from the National Health Interview Survey. BMC Public Health 12:520 [PubMed: 22788387]

11. Stanley S, Arriola KJ, Smith S, Hurlbert M, Ricci C, Escoffery C (2013) Reducing barriers to breast cancer care through Avon patient navigation programs. J Public Health Manag Pract. 19(5): 461-467. 10.1097/phh.0b013e318276e272 [PubMed: 23266756]

12. Raich PC, Whitley EM, Thorland W, Valverde P, Fairclough D (2012) Denver Patient Navigation Research Program. Patient navigation improves cancer diagnostic resolution: an individually randomized clinical trial in an underserved population. Cancer Epidemiol Biomarkers Prev 21(10): 1629-1638 [PubMed: 23045537]

13. Natale-Pereira Ana, Enard Kimberly R, Nevarez Lucinda, Jones Lovell A (2011) The role of patient navigators in eliminating health disparities. Cancer 117(15):3543-3552 [PubMed: 21780089]

14. Ekwueme DU, Gardner JG, Subramanian S, Tangka FK, Bapat B, Richardson LC (2008) Cost analysis of the National Breast and Cervical Cancer Early Detection Program: selected states, 2003 to 2004. Cancer 112(3):626-635 [PubMed: 18157831]

15. Hoerger TJ, Ekwueme DU, Miller JW et al. (2011) Estimated effects of the National Breast and Cervical Cancer Early Detection Program on breast cancer mortality. Am J Prev Med 40(4):397404. 10.1016/j.amepre.2010.12.017 [PubMed: 21406272]

16. Subramanian S, Ekwueme DU, Gardner JG, Trogdon J (2009) Developing and testing a costassessment tool for cancer screening programs. Am J Prev Med 37(3):242-247. 10.1016/j.amepre. 2009.06.002 [PubMed: 19666160]

17. Trogdon JG, Ekwueme DU, Subramanian S, Crouse W (2014) Economies of scale in federallyfunded state-organized public health programs: results from the National Breast and Cervical Cancer Early Detection Programs. Health Care Manag Sci 17:321-330 [PubMed: 24326873]

18. Rim S, Allaire B, Ekwueme D, Miller J, Subramanian S, Hall I, Hoerger T (2019) Costeffectiveness of breast cancer screening in the National Breast and Cervical Cancer Early Detection Program. Cancer Causes Control. 10.1007/s10552-019-01178-y

19. Cronin KA, Yu B, Krapcho M et al. (2005) Modeling the dissemination of mammography in the U.S. Cancer Causes Control 16:701-712 [PubMed: 16049809]

20. Plevritis SK, Salzman P, Sigal BM, Glynn P (2007) A natural history model of stage progression applied to breast cancer. Stat Med 26:581-595 [PubMed: 16598706]

21. Feuer EJ (2006) Modeling the impact of adjuvant therapy and screening mammography on U.S. breast cancer mortality between 1975 and 2000: introduction to the problem. J Natl Cancer Inst Monogr 36:2-6

22. Wells KJ, Battaglia TA, Dudley DJ et al. (2008) Patient navigation: state of the art or is it science? Cancer 113(8):1999-2010 [PubMed: 18780320]

23. Battaglia TA, Bak SM, Heeren T et al. (2012) Boston Patient Navigation Research Program: the impact of navigation on time to diagnostic resolution after abnormal cancer screening. Cancer Epidemiol Biomarkers Prev 21(10):1645-1654 [PubMed: 23045539]

24. Clark CR, Baril N, Kunicki M et al. (2009) Addressing social determinants of health to improve access to early breast cancer detection: results of the Boston REACH 2010 Breast and Cervical Cancer Coalition Women's Health Demonstration Project. J Womens Health 18(5):677-690

25. Markossian TW, Darnell JS, Calhoun EA (2012) Follow-up and timeliness after an abnormal cancer screening among under-served, urban women in a patient navigation program. Cancer Epidemiol Biomarkers Prev 21(10):1691-1700 [PubMed: 23045544]

26. Battaglia TA, Roloff K, Posner MA, Freund KM (2007) Improving follow-up to abnormal breast cancer screening in an urban population. A patient navigation intervention. Cancer 109(2 Suppl): 359-367 [PubMed: 17123275]

27. Ferrante JM, Chen PH, Kim S (2008) The effect of patient navigation on time to diagnosis, anxiety, and satisfaction in urban minority women with abnormal mammograms: a randomized controlled trial. J Urban Health 85(1):114-124 [PubMed: 17906931]

Cancer Causes Control. Author manuscript; available in PMC 2019 October 01. 
28. Miller JW, Hanson V, Johnson GD, Royalty JE, Richardson LC (2014) From cancer screening to treatment: service delivery and referral in the National Breast and Cervical Cancer Early Detection Program. Cancer 120:2549-2556. 10.1002/cncr.28823 [PubMed: 25099897]

29. Hoffman HJ, LaVerda NL, Young HA et al. (2012) Patient navigation significantly reduces delays in breast cancer diagnosis in the District of Columbia. Cancer Epidemiol Biomarkers Prev 21(10): 1655-1663 [PubMed: 23045540]

30. Freeman HP, Rodriguez RL (2011) History and principles of patient navigation. Cancer 117:35393542 [PubMed: 21780088]

31. Percac-Lima S, Ashburner JM, Bond B, Oo SA, Atlas SJ (2013) Decreasing disparities in breast cancer screening in refugee women using culturally tailored patient navigation. J Gen Intern Med 28(11):1463-1468 [PubMed: 23686510]

32. Percac-Lima S, Ashburner JM, McCarthy AM, Piawah S, Atlas SJ (2015) Patient navigation to improve follow-up of abnormal mammograms among disadvantaged women. J Womens Health (Larchmt) 24(2):138-143 [PubMed: 25522246]

33. Donelan K, Mailhot JR, Dutwin D et al. (2011) Patient perspectives of clinical care and patient navigation in follow-up of abnormal mammography. J Gen Intern Med 26(2):116-122 [PubMed: 20607432]

34. Molina Y, Kim S, Berrios N, Calhoun EA (2015) Medical mistrust and patient satisfaction with mammography: the mediating effects of perceived self-efficacy among navigated African American women. Health Expect 18(6):2941-2950 [PubMed: 25308749]

35. Levy AR, Bruen BK, Ku L (2012) Health Care Reform and Women's Insurance Coverage for Breast and Cervical Cancer Screening. [Erratum appears in Prev Chronic Dis 2012;9. http:// www.cdc.gov/pcd/issues/2012/12_0069e.htm. Prev Chronic Dis 9:120069 10.5888/pcd9.120069

36. Dohan D, Schrag D (2005) Using navigators to improve care of underserved patients: current practices and approaches. Cancer 104(4):848-855 [PubMed: 16010658] 


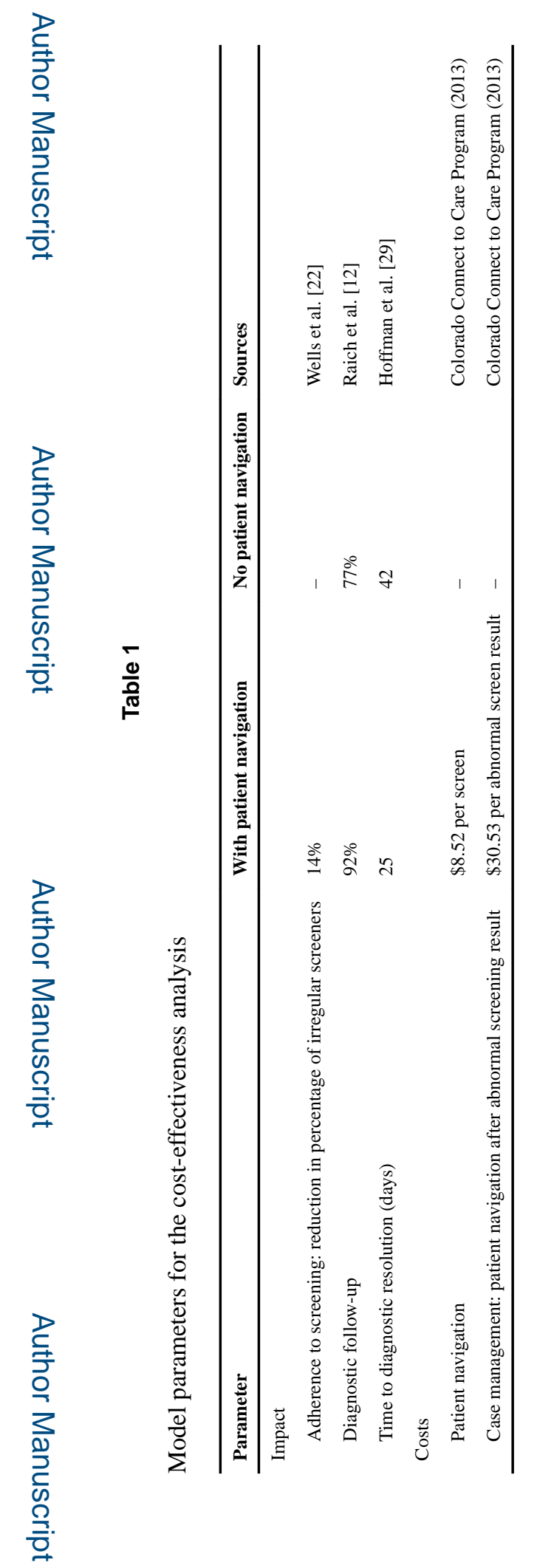

Cancer Causes Control. Author manuscript; available in PMC 2019 October 01. 


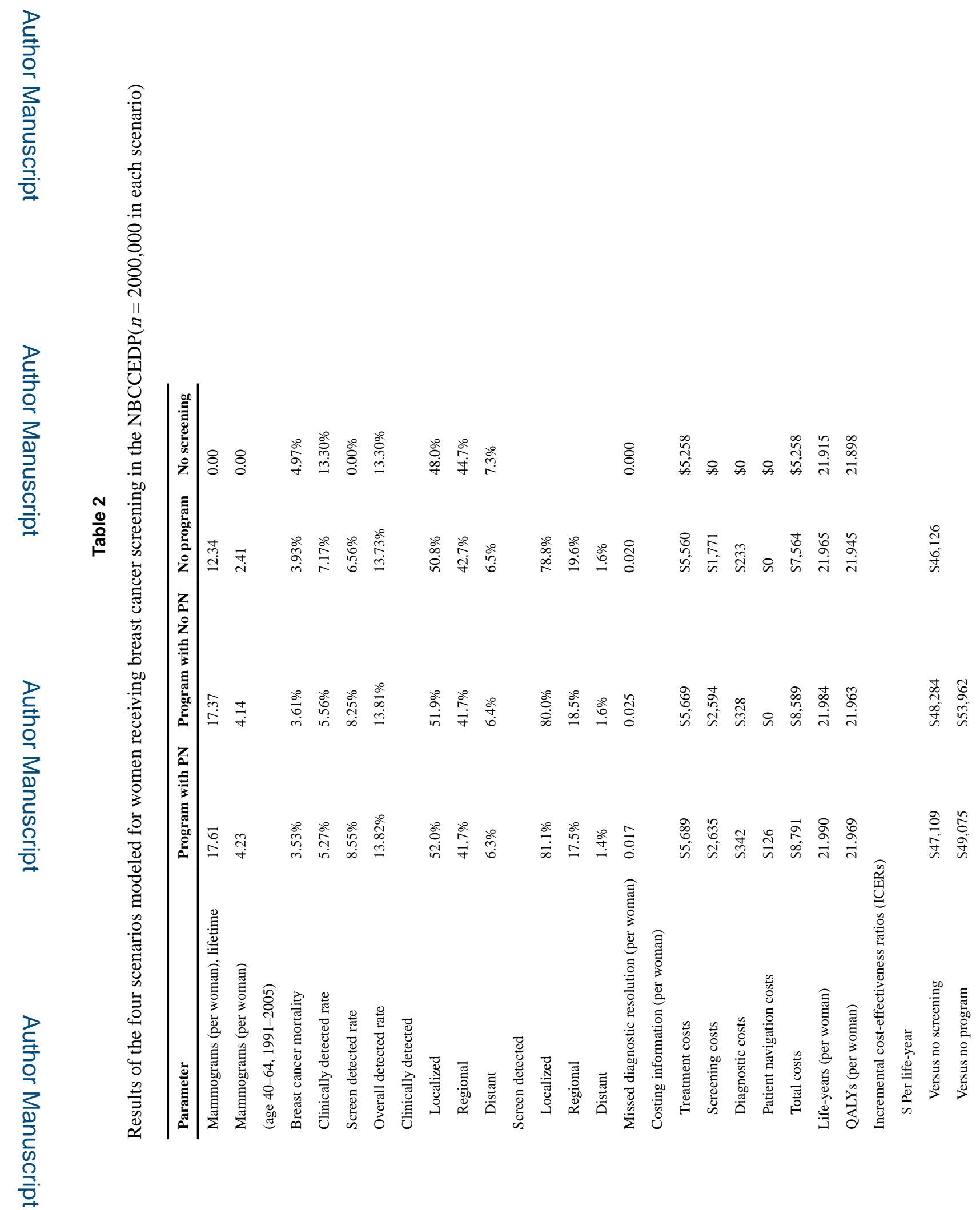

Cancer Causes Control. Author manuscript; available in PMC 2019 October 01. 
Allaire et al.

Page 12

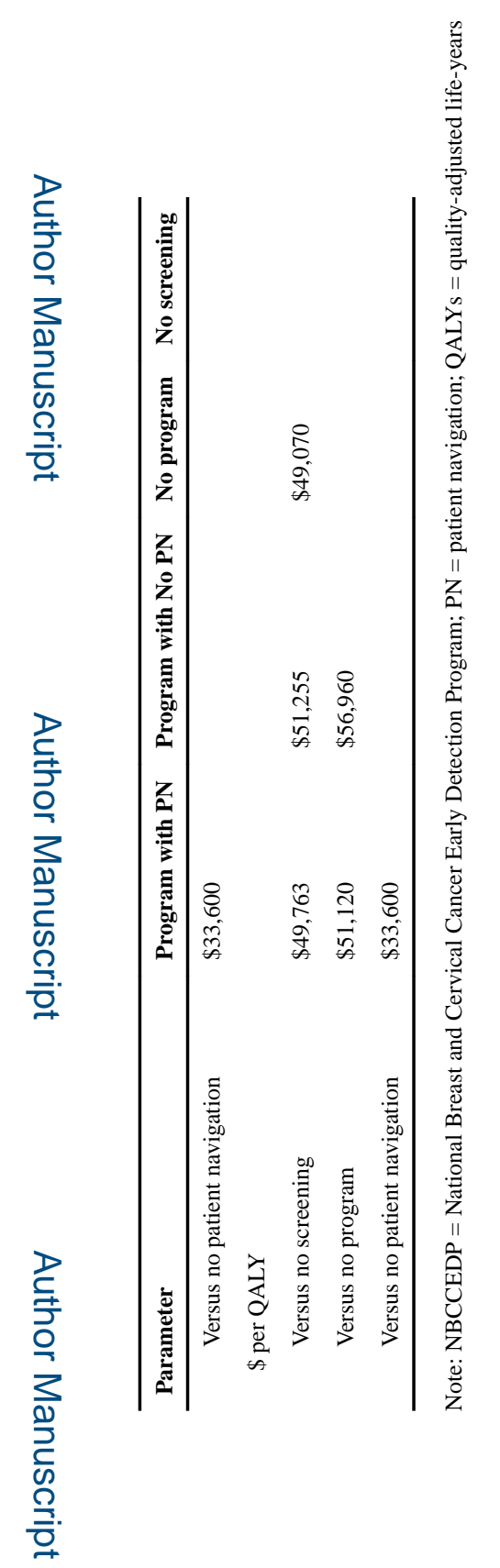

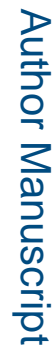

Cancer Causes Control. Author manuscript; available in PMC 2019 October 01. 